\title{
New Model for the Overall Transformation Kinetics of Bainite. Part 2: Validation
}

\author{
María Jesús Santofimia*, Francisca G. Caballero, Carlos Capdevila, \\ Carlos García-Mateo and Carlos García de Andrés \\ Materalia Research Group, Department of Physical Metallurgy, Centro Nacional de Investigaciones Metalúrgicas (CENIM), \\ Consejo Superior de Investigaciones Científicas (CSIC), Avda. Gregorio del Amo, 8. E-28040 Madrid, Spain
}

\begin{abstract}
A new model for the overall kinetics of the bainite transformation has been validated experimentally. The new model, presented in the 1st part of this work, is based in the displacive mechanism for bainite transformation. Thus, the bainite transformation kinetics of three medium carbon-high silicon steels has been studied. Results show that the model, which does not consider the effect of carbide precipitation in the bainite kinetics, predicts with a high degree of agreement the time evolution of bainitic ferrite volume fraction, even when lower bainite is present at the microstructure. Data from two additional medium carbon-high silicon steels, frequently reported in the literature, have been also used for reinforcing the validation, obtaining, again, a high agreement between the kinetic results for bainite transformation predicted by the model and those obtained experimentally. [doi:10.2320/matertrans.47.2473]
\end{abstract}

(Received June 5, 2006; Accepted August 15, 2006; Published October 15, 2006)

Keywords: bainite, transformation kinetics, steels

\section{Introduction}

In the first part of this study, a new model for the kinetics of the bainite transformation has been proposed. The model is based on the displacive mechanism for bainite transformation. An important characteristic of this new model is the complete separation between the kinetics of both nucleation events of bainitic ferrite subunits, in austenite grain boundaries and at subunits previously formed. This distinction is based in a geometrical conception of the development of the transformation and has led to the elimination of the autocatalysis factor, an obscure parameter used in former kinetics models. The model correctly predicts the effect of carbon and other alloying elements such manganese and cobalt on the transformation kinetics, as it was shown in the first part of this work.

The precipitation of cementite between the subunits of bainitic ferrite during bainitic transformation can be suppressed by alloying the steel with about 1.5 mass $\%$ silicon, which has very low solubility in cementite and greatly retards its growth from austenite. ${ }^{1-3)}$ The carbon that is rejected from the bainitic ferrite enriches the residual austenite, thereby stabilising it down to room temperature. Consequently, an isothermal transformation in the range of the bainite transformation leads to a microstructure consisting of bainitic ferrite separated by carbon-enriched regions of austenite. Since the cementite precipitation between the plates of bainitic ferrite plates has not been considered in the modelling, steels with high silicon content are most adequate for the validation of the proposed model. However, cementite precipitation within bainitic ferrite plates (lower bainite), that can occur even in high silicon steels, has not been considered in the model. In this sense, the study of lower bainite kinetics

*Present address: Netherlands Institute for Metals Research, Mekelweg 2, 2628 CD Delft, Netherlands. Department of Materials Science and Engineering, Delft University of Technology, Mekelweg 2, 2628 CD, Delft, Netherlands. is interesting in order to evaluate the reliability of the model predictions in the event of lower bainite formation.

It has been shown in the 1st part of the study that if no other reaction interacts with the successive nucleation and growth of subunits of bainitic ferrite, the incomplete reaction phenomenon, by means of the $T_{0}^{\prime}$ curve, provides a method for the estimation of the maximum volume fraction of bainitic ferrite that can be formed, at a given temperature, in a steel, $v_{\alpha_{b}-\max }$. Considering a steel with a nominal carbon content $\bar{x}$, the value of $v_{\alpha_{b}-\max }$ is given by:

$$
v_{\alpha_{b}-\max }=\frac{x_{T_{0}^{\prime}}-\bar{x}}{x_{T_{0}^{\prime}}-x_{\alpha_{b}}}
$$

where $x_{T_{0}^{\prime}}$ is the carbon content of the residual austenite, given by the $T_{0}^{\prime}$ curve, $x_{\alpha_{b}}$ stands for the carbon content of the bainitic ferrite, given by the equilibrium value.

The work presented in this part of the study, deals with the experimental validation of the model. With this aim, the isothermal kinetics of the bainite transformation of three medium carbon-high silicon steels have been characterised and the results thus obtained are compared with the model predictions. Moreover, data from two additional medium carbon-high silicon steels, widely used for the evaluation of kinetic models in the literature are also used to extend the range of validation of the proposed model.

\section{Experimental Procedure}

The chemical composition of the studied steels is given in Table 1 . The grades were supplied as $12 \mathrm{~mm}$ hot rolled strips. Dilatometric analysis and heat treatments were carried out using an Adamel Lhomargy DT1000 high-resolution dilatometer on cylindrical test pieces of $3 \mathrm{~mm}$ in diameter and $12 \mathrm{~mm}$ in length. The dimensional variations in the specimens are transmited via an amorphous silica pushrod and measured by a linear variable differential transformer (LVDT) in a gas-tight enclosure, enabling to monitor the isothermal transformations to bainite. ${ }^{4)}$ The temperature is 
Table 1 Chemical compositions of the studied steels in mass $\%$.

\begin{tabular}{ccccccccc}
\hline Steel & $\mathrm{C}$ & $\mathrm{Si}$ & $\mathrm{Mn}$ & $\mathrm{Ni}$ & $\mathrm{Cr}$ & $\mathrm{Mo}$ & $\mathrm{Co}$ & $\mathrm{Al}$ \\
\hline $\mathrm{B}$ & 0.29 & 1.48 & 2.06 & - & 0.43 & 0.27 & - & - \\
$\mathrm{C}$ & 0.29 & 1.45 & 1.97 & - & 0.95 & 0.26 & 0.67 & - \\
$\mathrm{D}$ & 0.31 & 1.40 & 1.97 & 1.70 & 1.41 & 0.26 & 1.13 & 1.01 \\
\hline
\end{tabular}

Table 2 Austenitisation conditions and prior austenite grain size by the use of the maximum distance between sides of a three dimensional tetrakahidecahedra.

\begin{tabular}{cccc}
\hline Steel & $T_{\gamma} /{ }^{\circ} \mathrm{C}$ & $t_{\gamma} / \mathrm{s}$ & $D_{c} / \mu \mathrm{m}$ \\
\hline B & 925 & 300 & $12 \pm 5$ \\
C & 925 & 300 & $14 \pm 6$ \\
D & 925 & 300 & $15 \pm 7$ \\
\hline
\end{tabular}

measured with a $0.1 \mathrm{~mm}$ diameter chromel-alumel (type $\mathrm{K}$ ) thermocouple spot welded to the specimen.

In order to obtain fully decomposition of austenite into bainite, samples were subjected to an austenitisation at $925^{\circ} \mathrm{C}$ during five minutes followed by rapid cooling to the isothermal transformation temperature, between 200 and $600^{\circ} \mathrm{C}$, for different periods of times before quenching to room temperature. Dilatometry results were complemented with metallography observation in Light Optical Microscopy and Scanning Electron Microscope (JEOL JSM-6500F filed emission gun operating at $7 \mathrm{kV}$ ). Sample preparation was made following standard techniques and the microstucture was revealed by etching with $2 \%$ nital.

The heating and cooling devices of this dilatometer have been also used to study previously the austenitisation condition of these steels. In this sense, cylindrical dilatometric test pieces of $3 \mathrm{~mm}$ in diameter and $12 \mathrm{~mm}$ in length were used to reveal grain boundaries by the thermal etching. ${ }^{5)}$ For this purpose, a surface $2 \mathrm{~mm}$ in width was generated along the longitudinal axis of samples by polishing and finishing with $1 \mu \mathrm{m}$ diamond paste. Later on, samples were austenitised in vacuum $(>1 \mathrm{~Pa})$ at $925^{\circ} \mathrm{C}$ for 300 seconds. Subsequently samples were cooled down to room temperature at $1{ }^{\circ} \mathrm{C} / \mathrm{s}$. These samples do not require metallographic preparation after heat treatment; the prior austenite grain boundaries are revealed without chemical etching. The average austenite grain size was measured using an image analyser. Table 2 shows the resulting austenitisation conditions and the measurements of the austenite grain size, characterised by the use of the distance between opposite sides of three dimensional tetrakahidecahedra. ${ }^{6-8)}$

The volume fraction of bainitic ferrite was estimated by a systematic manual point-counting procedure on optical and scanning electron micrographs at low magnification. ${ }^{9)}$ A grid superimposed on the microstructure provides, after a suitable number of placements, an unbiased statistical estimation of the volume fraction of bainitic ferrite. Stereological errors for the values of the volume fraction of bainitic ferrite correspond to the standard deviation of the measurement. In these steels, where the cementite precipitation from residual austenite is inhibited by the judicious use of silicon, the metallographic measurements of the volume fraction bainitic ferrite were corrected taking into account that the bainitic sheaves contain approximately $15 \%$ of thin films of retained austenite. ${ }^{10)}$

The martensite start temperature $\left(M_{s}\right)$ of each steel was measured by dilatometry, by heating to $925^{\circ} \mathrm{C}$ and then rapidly cooled. Each dilatometric test was performed twice. The formation of martensite during cooling was detected by monitoring the fractional change in dilatation with temperature.

Finally, quantitative X-ray analysis was used to determine the total volume fraction of retained austenite in the steels after the completion of the bainite transformation. For this purpose, $11 \times 5 \times 2 \mathrm{~mm}^{3}$ samples were machined. After grinding and final polishing using $1 \mu \mathrm{m}$ diamond paste, the samples were etched to obtain an undeformed surface. They were then step-scanned in a SIEMENS D 5000 X-ray diffractometer using unfiltered $\mathrm{Cu} K_{\alpha}$ radiation. The scanning speed $(2 \theta)$ was less than 0.3 degree $/ \mathrm{min}$. The machine was operated at $40 \mathrm{kV}$ and $30 \mathrm{~mA}$. The retained austenite content was calculated from the integrated intensities of (200), (220) and (311) austenite peaks and those of (002), (112) and (022) planes of ferrite. ${ }^{11)}$ Using three peaks from each phase avoids biasing the results due to any crystallographic texture in the samples. ${ }^{12)}$ The carbon concentration in the austenite was estimated by using the lattice parameters of the retained austenite. ${ }^{13)}$

\section{Results and Discussion}

Silicon can avoid the precipitation of cementite between the plates of bainitic ferrite, but it does not have any effect on the formation of cementite inside the ferrite plates. Therefore, the formation of lower bainite was not inhibited in these steels. In this work, the kinetics of the bainite transformation has been characterised in the three steels between the $M_{S}$ and the $B_{S}$ temperature, i.e., including the formation of upper (carbide free) and/or lower bainite (carbides within ferrite). Thus, the model ability to predict the bainite transformation kinetics, even in the case of formation of lower bainite, can be evaluated. This fact is interesting, taking into account that the cementite precipitation theory has not been included in the model.

Metallographic examination by LOM and SEM allowed determining the bainite start temperature $\left(B_{S}\right)$ and lower bainite start temperature $\left(L B_{S}\right)$. Between both $B_{S}$ and $L B_{S}$ temperatures, only upper bainite is formed. Figure 1 shows examples of lower bainite microstructure in steels B, C and D after isothermal transformation at $325^{\circ} \mathrm{C}$. Particles of cementite are easily identified inside the bainitic ferrite plates. Experimentally measured values of $M_{S}, B_{S}$ and $L B_{S}$ for all steel grades are displayed in Table 3.

As a first stage on the validation of the model with experimental results, the applicability of the $T_{0}^{\prime}$ curve for the determination of the residual austenite carbon content at the end of the bainitic transformation is evaluated. Figure 2 shows a comparison between the experimental values of the carbon content of the retained austenite in the steels B, C and $\mathrm{D}$ and the predicted $T_{0}, T_{0}^{\prime}$ and equilibrium $A e_{3}$ curves. It is observed that measured retained austenite carbon content, $x_{\gamma}$, is close to the $T_{0}$ and $T_{0}^{\prime}$ curves, which is in agreement with the incomplete reaction phenomenon characteristic of the 

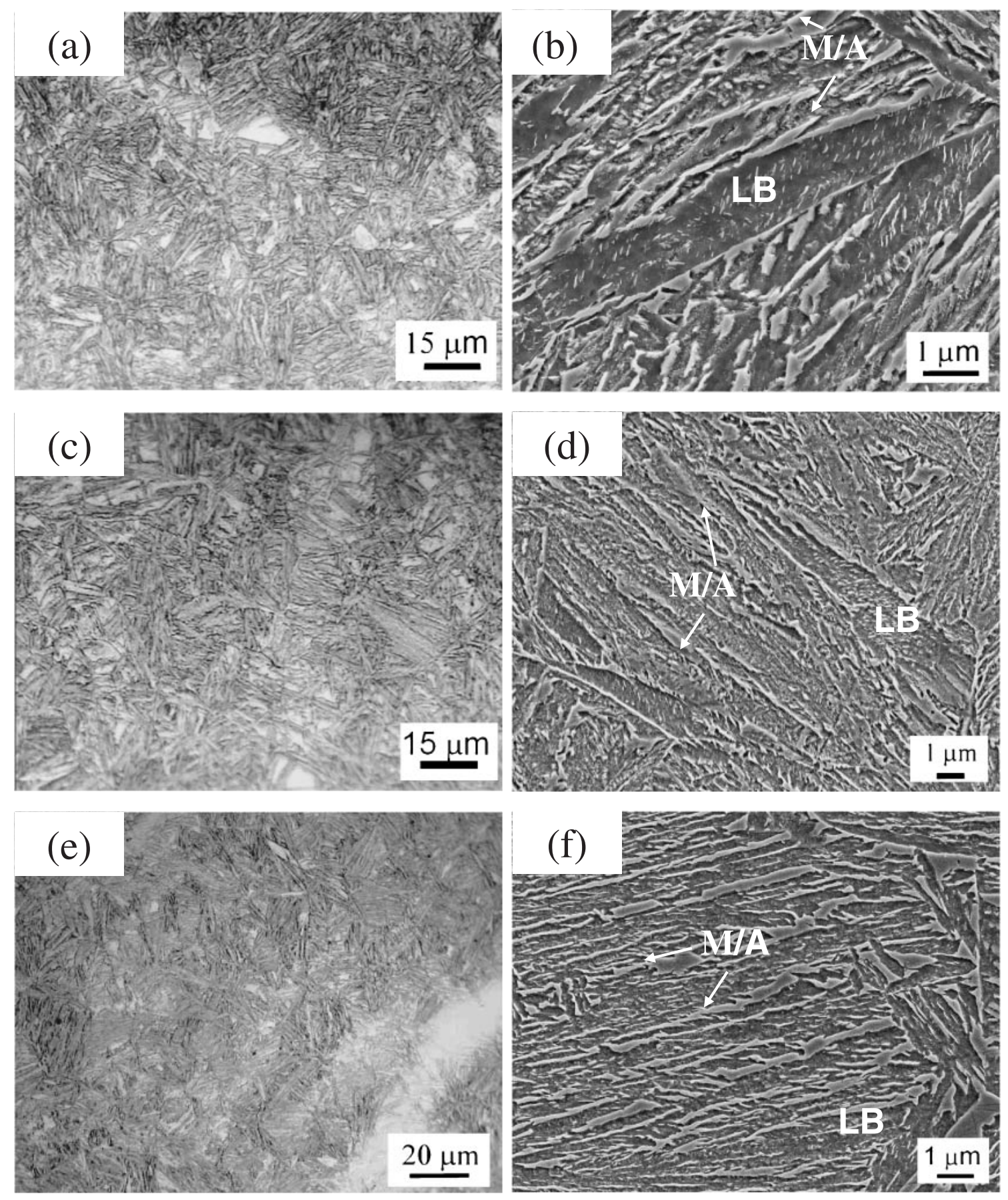

Fig. 1 Optical and scanning micrographs of microstructures obtained by isothermal transformation at $325^{\circ} \mathrm{C}$ for (a,b) $1800 \mathrm{~s}$ in steel B, $(\mathrm{c}, \mathrm{d}) 1800 \mathrm{~s}$ in steel C and (e,f) $7200 \mathrm{~s}$ in steel D. LB means lower bainite and M/A, martensite and/or retained austenite.

Table 3 Experimental values of $B_{S}, L B_{S}$ and $M_{S}$ temperatures.

\begin{tabular}{cccc}
\hline Steel & $M_{S} /{ }^{\circ} \mathrm{C}$ & $L B_{S} /{ }^{\circ} \mathrm{C}$ & $B_{S} /{ }^{\circ} \mathrm{C}$ \\
\hline $\mathrm{B}$ & 300 & 400 & 450 \\
$\mathrm{C}$ & 300 & 400 & 400 \\
$\mathrm{D}$ & 300 & 375 & 400 \\
\hline
\end{tabular}

diffusionless mechanism for bainite transformation. It is worth to highlight the fact that the $T_{0}^{\prime}$ curve generally provides a slight underestimation of the experimental values. This is understood if the nature of bainitic transformation is taken into account. The consideration of the displacive growth mechanism of the subunits leads to a non-homogeneous distribution of carbon in the residual austenite during the bainitic transformation. ${ }^{14)}$ The films of residual austenite, between the subunits of bainitic ferrite in a sheaf, are isolated by the surrounding bainite and, therefore can achieve carbon concentration levels far in excess of those expected assuming a homogenous distribution. On the contrary, blocks of untransformed residual austenite between the sheaves of bainite are correspondingly less carbon enriched due to the low proportion of carbon received during the bainite transformation. After the final cooling following the transformation, the blocks of residual austenite tend to transform to martensite, whereas the carbon enriched films of residual 

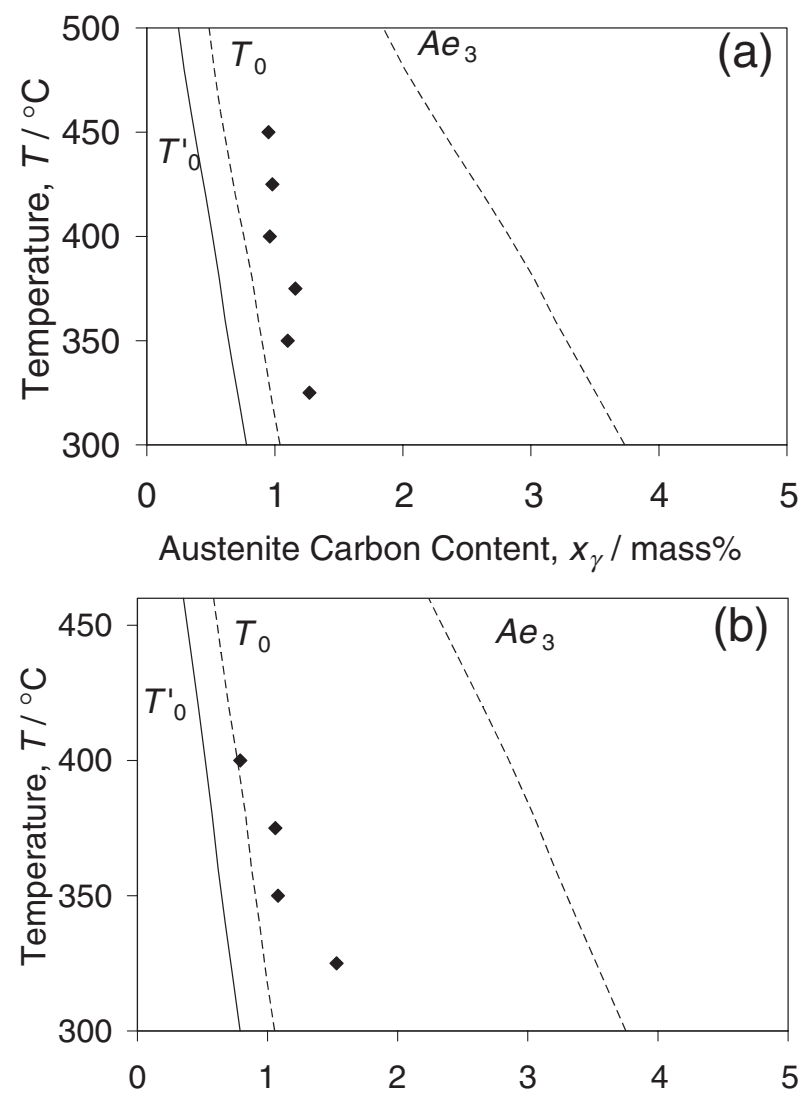

Austenite Carbon Content, $x \gamma /$ mass $\%$

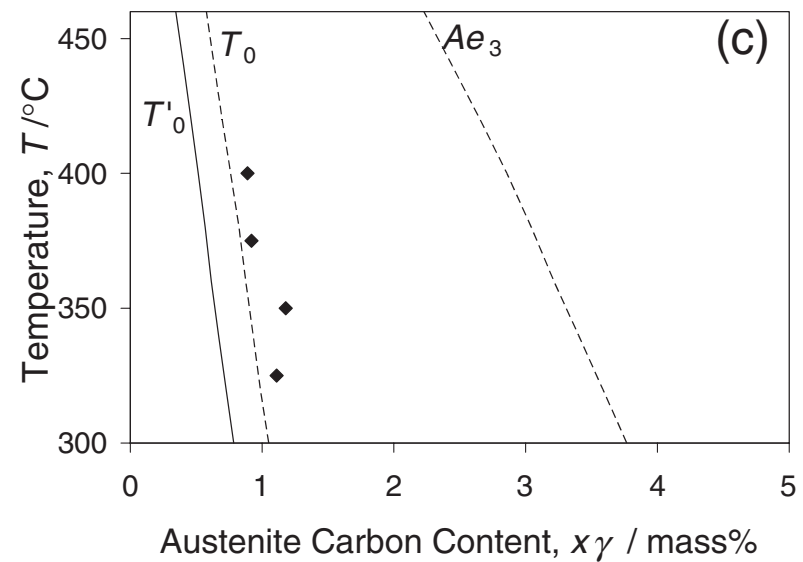

Fig. 2 Carbon content of the retained austenite at the end of the bainite transformation and comparison with the calculated $T_{0}^{\prime}$ curve for steels (a) B, (b) C and (c) D.

austenite are retained. Since the X-ray measurements provide the carbon content of the untransformed austenite, it is reasonable to assume that these measurements are an overestimation of the mean carbon content of the residual austenite at the end of the bainite transformation. Having in mind this fact, Figure 2 demonstrates that the use of the $T_{0}^{\prime}$ curve for the estimation of the carbon content of the residual austenite in the proposed model is adequate.

On the other hand, the applicability of eq. (1) for the calculation of the maximum volume fraction of bainitic ferrite that can be formed at the end of the transformation, $v_{\alpha_{b}-\max }$, in the particular case of the steels $\mathrm{B}, \mathrm{C}$ and $\mathrm{D}$, is studied. Figure 3 shows the correlation between the calcu-

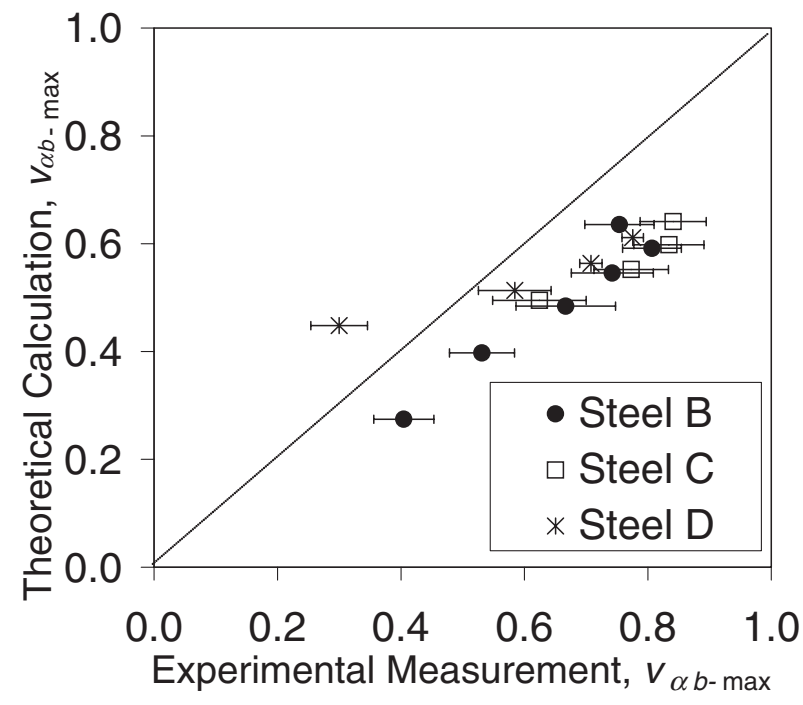

Fig. 3 Degree of agreement of calculated and experimental maximum volume fractions of bainitic ferrite for the steels B, C and D.

lated values of $v_{\alpha_{b}-\max }$ and the experimental results. In general it is observed, that predicted $v_{\alpha_{b}-\max }$ values are lower than the experimental ones. This difference can also be explained in terms of the non-uniform distribution of carbon in residual austenite, ${ }^{14)}$ therefore, higher $v_{\alpha_{b}-\max }$ should be expected from this poor carbon austenite than in the case of assuming a homogeneous carbon distribution. Moreover, there is another argument that supports the fact that theoretical fractions of ferrite are lower than those obtained experimentally: the carbon consumption during carbide precipitation within bainitic ferrite plates, at temperatures lower than $L B_{S}$. In lower bainite, carbide precipitation acts as sink of carbon that are not considered in the eq. (1), allowing a smaller carbon enrichment of the residual austenite and, consequently, a higher value of the maximum volume fraction of bainitic ferrite. Therefore, the applicability of the eq. (1) is at some point limited because it does not take into account all the factors that affect the value of $v_{\alpha_{b}-\max }$. Thus, the model would not be able to predict with absolute reliability the value of the volume fraction of bainitic ferrite formed at each transformation time if eq. (1) was used in these steels. Since the objective of this work is the validation of the kinetic results predicted by the model, the experimental value of $v_{\alpha_{b}-\max }$ has been introduced as an input for the validation in order to eliminate the error associated to the use of eq. (1) in the predictions. By this procedure, kinetics predictions of the model are evaluated without a possible lack of precision in the theoretical estimation of $v_{\alpha_{b}-\max }$. As it has been proved that the $T_{0}^{\prime}$ curve adequately predicts the carbon content of the residual austenite at the end of the transformation, this curve is used for its evaluation.

Figure 4 summarises the bainite transformation kinetics predictions and experimental measurements for steels B, C and D. It is also shown the degree of agreement between predictions for the volume fraction of bainitic ferrite formed and the experimental measurements. Moreover, Table 4 shows the values of the slope and the $R^{2}$ coefficient of a linear fit crossing the origin of the coordinates corresponding to the points of the representation shown in the Figures 4(b), 

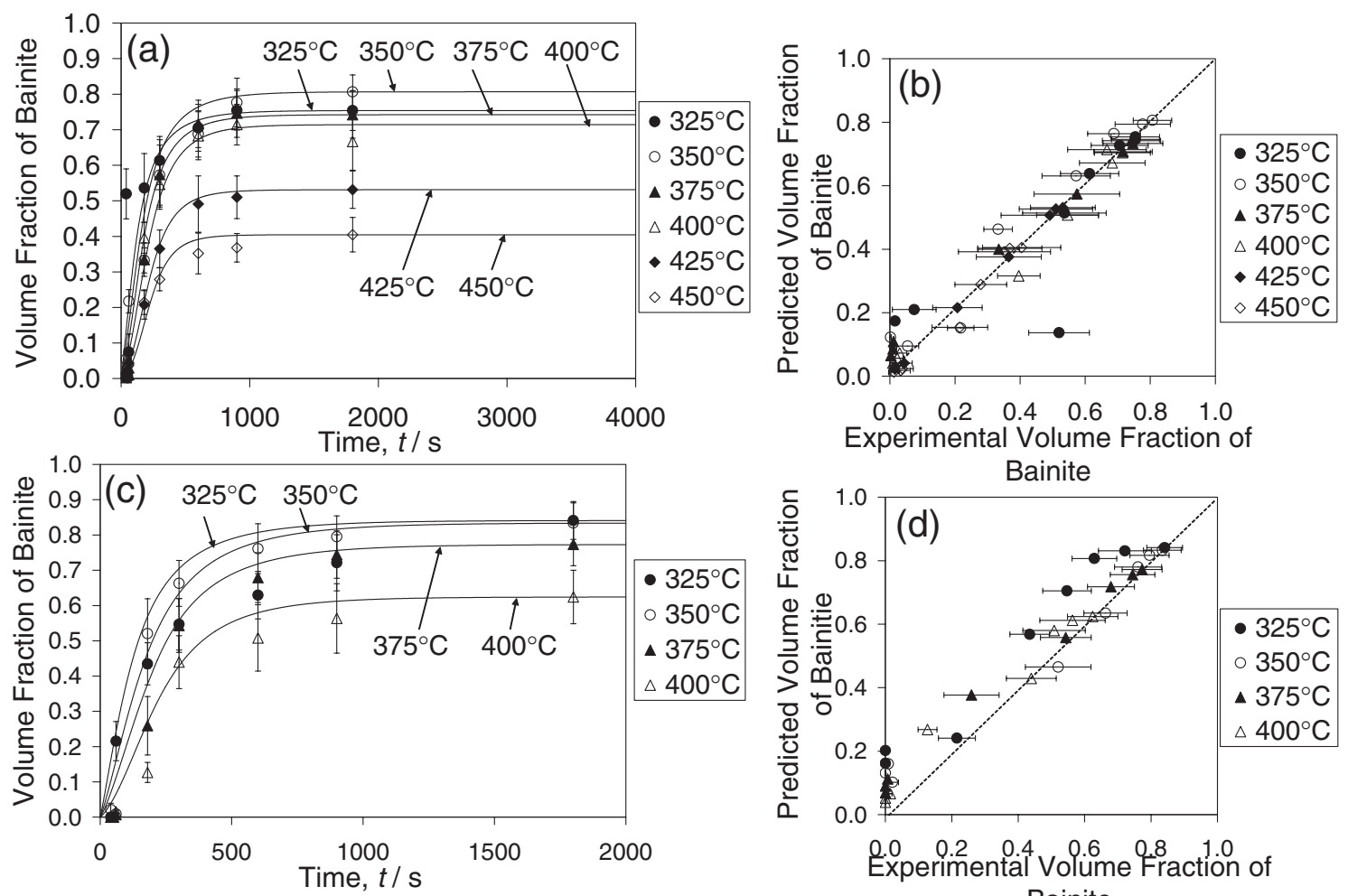

Bainite
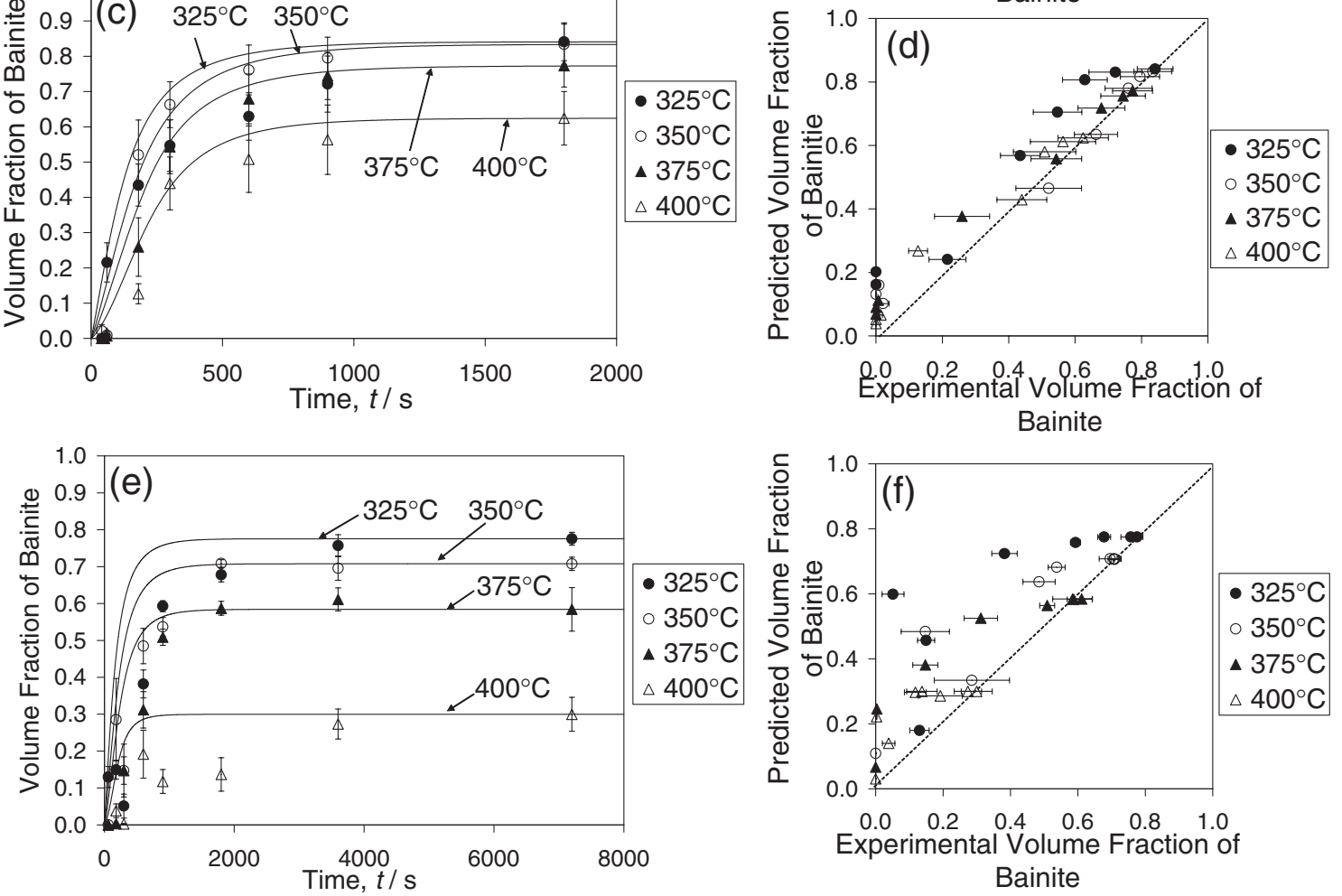

Fig. 4 Comparison of calculated and experimentally determined isothermal transformations curves and degree of agreement of predicted and experimental volume fractions of bainitic ferrite in steels $(a, b) B,(c, d) C$ and $(e, f) D$.

Table 4 Values of the slope and $R^{2}$ coefficient of a linear fit crossing the origin of coordinates corresponding to a representation of the volume fraction of bainitic ferrite predicted versus experimental in alloys B, C and $\mathrm{D}$.

\begin{tabular}{ccc}
\hline Steel & Slope & $R^{2}$ \\
\hline B & 0.99 & 0.98 \\
C & 1.06 & 0.91 \\
D & 1.13 & 0.46 \\
\hline
\end{tabular}

4(d) and 4(f). It can be observed that the model reproduces with excellent agreement the experimental curves of the isothermal transformation in steels $\mathrm{B}$ and $\mathrm{C}$, including at the temperatures at which lower bainite forms. Moreover, the obtained values of $R^{2}$ indicate a negligible dispersion of the results around the linear fit. This result suggests that the kinetics of the cementite precipitation inside the plates of lower bainite does not significantly affect the overall transformation kinetics and, consequently, formation of lower bainite does not seem to be an obstacle in the applicability of the proposed model. However, in the case of the steel D, the predictions correspond to a faster kinetic of transformation than that experimentally observed. A value of $R^{2}$ equal to 0.46 indicates that the points in Fig. 4(f) do not obey a linear fit. In this case, it seems that the complex alloying content of this steel has not allowed an adequate prediction with the proposed model. Possible combined effects from alloying elements on phase transformations are not taken into account.

As it was anticipated, two more steels, widely employed in the literature for similar studies, ${ }^{15-20)}$ have been also used to reinforce the validation of the model. Their chemical composition and the measured austenite grain size after an austenitisation at $1000^{\circ} \mathrm{C}$ for $300 \mathrm{~s}$ are shown in the Table 5 .

In order to follow a coherency between these predictions and those made previously for the steels B, C and D, the validation of the model has been also carried out introducing the experimental value of $v_{\alpha_{b}-\max }$ as an input. By contrast with former case, calculations of $v_{\alpha_{b}-\max }$ by using the eq. (1) 
Table 5 Chemical composition and austenite grain size of steels selected from the literature. ${ }^{15-20)}$

\begin{tabular}{lcccccccr}
\hline \multirow{2}{*}{ Steel } & \multicolumn{7}{c}{ Chemical composition in mass\% } & mean linear intercept $(\mu \mathrm{m})$ \\
\cline { 2 - 7 } & $\mathrm{C}$ & $\mathrm{Si}$ & $\mathrm{Mn}$ & $\mathrm{Ni}$ & $\mathrm{Mo}$ & $\mathrm{Cr}$ & $\mathrm{V}$ \\
\hline FeNiSiC & 0.39 & 2.05 & - & 4.08 & - & - & - \\
$300 \mathrm{M}$ & 0.44 & 1.74 & 0.67 & 1.85 & 0.83 & 0.39 & 0.09 \\
\hline
\end{tabular}
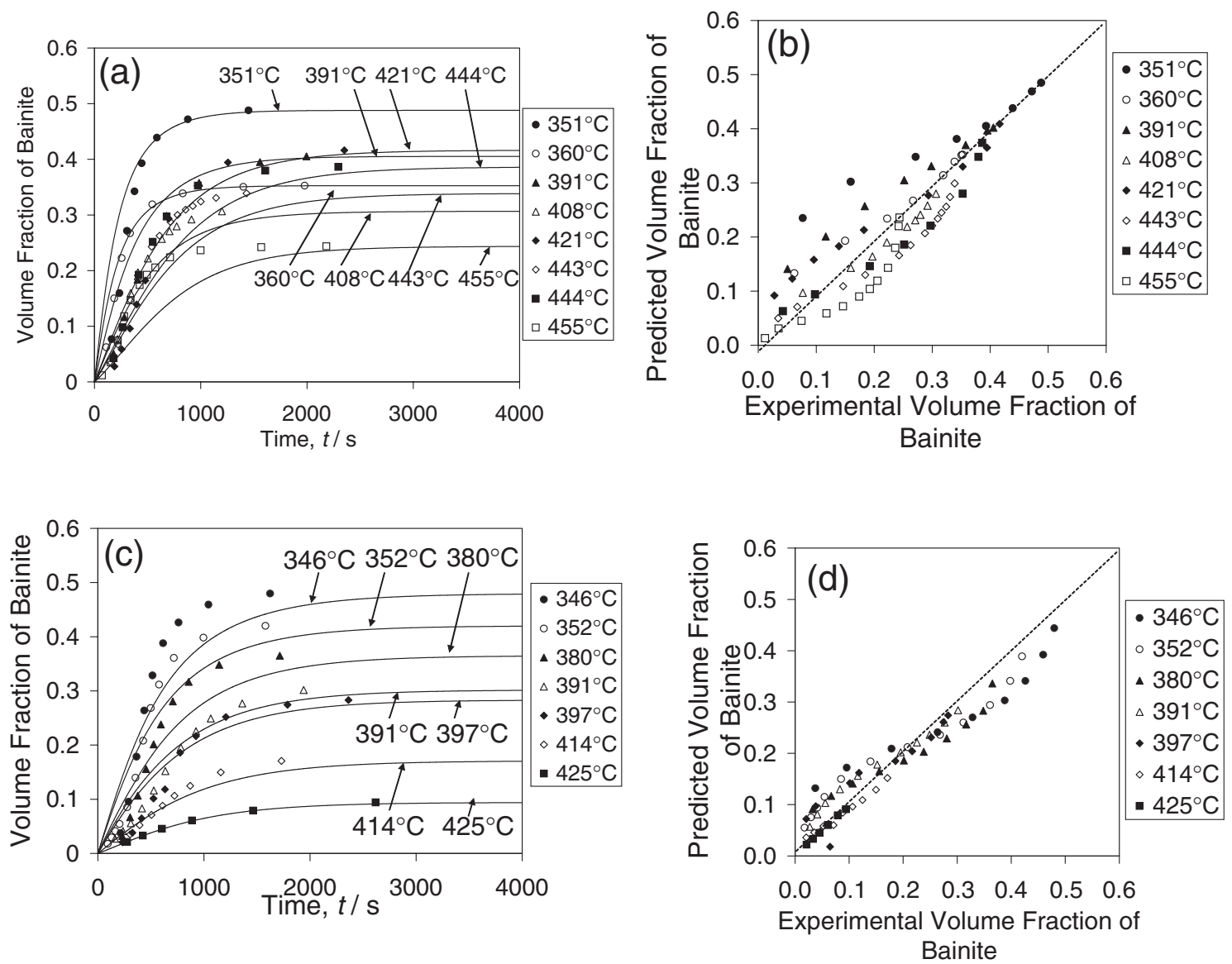

Fig. 5 Comparison of calculated and experimentally determined isothermal transformations curves and degree of agreement of predicted and experimental volume fraction of bainitic ferrite in steels (a,b) FeNiSiC and (c,d) 300M.

in the steels FeNiSiC and 300M, are accurate and the use of their corresponding experimental values would not imply big changes in the predictions. ${ }^{20)}$

Predictions for the bainite transformation kinetics along with experimental measurements are shown in Figs. 5(a) and $5(c)$, where it is observed that the calculated curves fits very accurately the experimental points. Likewise, the agreement between the calculated and experimental volume fraction of bainitic ferrite, showed in Figs. 5(b) and 5(d), is excellent for both steels. The resultant slopes correspond to 0.94 and 0.91 in the steels FeNiSiC and $300 \mathrm{M}$, respectively, which are values very near to the ideal value of 1 . Using these linear fits, the obtained value of $R^{2}$ in the steel FeNiSiC is 0.74 , indicating a slight dispersion of the values of the fit, and 0.87 in the case of $300 \mathrm{M}$, which corresponds to a higher alignment of the points.

\section{Conclusion}

A new model for the isothermal kinetics of bainite transformation, based on the displacive mechanism of transformation has been validated. With this aim, the kinetics of the bainite transformation in three medium carbon-high silicon steels has been studied in detail. The measurements of retained austenite carbon content in these steels obey with an acceptable agreement the criterion given by the $T_{0}^{\prime}$ curve, although the maximum volume fraction of bainitic ferrite calculated at every transformation temperature is not completely well predicted using these considerations. The reasons can be found in the non-homogeneous distribution of carbon in the residual austenite and in the carbide precipitation during lower bainite formation. Therefore, the $T_{0}^{\prime}$ curve has been used for the modelling of the carbon content of the residual austenite. However, it has been found more adequate the use of the experimental values of the maximum volume fraction of bainitic ferrite as inputs in the validation of the model. Results have shown that the model predicts with a high degree of accuracy the evolution of the volume fraction of bainitic ferrite with time. The same criteria have been used in the validation with the results of 
two additional medium carbon-high silicon steels frequently used in the literature. Likewise, there is an excellent agreement between the kinetic of the bainite transformation predicted by the model and the experimental measurements.

\section{Acknowledgement}

The authors acknowledge financial support from the European Coal and Steel Community (ECSC agreement number 7210-PR/345) and the Spanish Ministerio de Ciencia y Tecnología (Project-MAT 2002-10812 E). C. GarciaMateo would like to thank Spanish Ministerio de Ciencia y Tecnología for the financial support in the form of a Ramón y Cajal contract (RyC 2004 Program).

\section{REFERENCES}

1) S. W. Owen: Trans. ASM 46 (1954) 812-829.

2) H. K. D. H. Bhadeshia and D. V. Edmonds: Metall. Trans. A 10 (1979) 895-907.

3) H. K. D. H. Bhadeshia, M. Lord and L. S.-E. Svensson: Proceeding of Int. Conf. Joining \& Welding Solutions to Industrial Problems, (JWRI, Osaka University, 2003) pp. 43-52.

4) C. García de Andrés, F. G. Caballero, C. Capdevila and L. F. Álvarez: Mater. Charact. 48 (2002) 101-111.
5) C. García de Andrés, M. J. Bartolomé, C. Capdevila, D. San Martín, F. G. Caballero, V. López and J. Vara: Rev. Met. Madrid 37 (2001) 528-539.

6) J. W. Cahn: Acta Metall. 4 (1956) 572-575.

7) F. G. Caballero, C. Capdevila and C. García de Andrés: ISIJ Int. 41 (2001) 1093-1102.

8) D. San Martín: Ph.D. Thesis, (CENIM-CSIC and Universidad Complutense de Madrid, Madrid, Spain, 2003).

9) G. F. Vander Voort: Metallography. Principles and Practice, (McGraw-Hill Book Company, New York, U.S.A., 1984) pp. 426-428.

10) H. K. D. H. Bhadeshia and D. V. Edmonds: Metal Sci. 17 (1983) 411419.

11) J. Durnin and K. A. Ridal: J. Iron Steel Inst. 206 (1968) 60-67.

12) M. J. Dickson: J. Appl. Cryst. 2 (1969) 176-180.

13) D. J. Dyson and B. Holmes: J. Iron Steel Inst. 208 (1970) 469-474.

14) G. I. Rees and H. K. D. H. Bhadeshia: Mater. Sci. Technol. 8 (1992) 994-996.

15) H. K. D. H. Bhadeshia: J. Phys. IV 43 (1982) 443-448.

16) G. I. Rees: Ph. Thesis, (University of Cambridge, Cambridge, UK, 1991).

17) G. I. Rees and H. K. D. H. Bhadeshia: Mater. Sci. Technol. 8 (1992) 985-993.

18) S. B. Singh: Ph.D. Thesis, (University of Cambridge, Cambridge, UK, 1998).

19) P. Opdenacker: Ph.D. Thesis, (University of Cambridge, Cambridge, UK, 2001).

20) H. Matsuda and H. K. D. H. Bhadeshia: Proc. R. Soc. London A 460 (2004) 1707-1722. 